\title{
MODIFIED LIMITED MEMORY BFGS METHOD WITH NONMONOTONE LINE SEARCH FOR UNCONSTRAINED OPTIMIZATION
}

\author{
Gonglin Yuan, Zengxin Wei, and Yanlin Wu
}

\begin{abstract}
In this paper, we propose two limited memory BFGS algorithms with a nonmonotone line search technique for unconstrained optimization problems. The global convergence of the given methods will be established under suitable conditions. Numerical results show that the presented algorithms are more competitive than the normal BFGS method.
\end{abstract}

\section{Introduction}

Consider the following unconstrained optimization problem

$$
\min _{x \in \mathbb{R}^{n}} f(x),
$$

where $f: \mathbb{R}^{n} \rightarrow \mathbb{R}$ is continuously differentiable. The line search method is one of the most numerical method, which is defined by

$$
x_{k+1}=x_{k}+\alpha_{k} d_{k}, k=0,1,2, \ldots,
$$

where $\alpha_{k}$ that is determined by a line search is the steplength, and $d_{k}$ which determines different line search methods [35, 36, 37, 39, 40, 43, 44, 45, 46, 48, 50] is a search direction of $f$ at $x_{k}$.

One of the most effective methods for unconstrained optimization (1.1) is Newton method. It normally requires a fewest number of function evaluations, and is very good at handling ill-conditioning. However, its efficiency largely depends on the possibility to efficiently solve a linear system which arises when computing the search $d_{k}$ at each iteration

$$
G\left(x_{k}\right) d_{k}=-g\left(x_{k}\right)
$$

Received July 17, 2008

2000 Mathematics Subject Classification. 65H10, 65K05, 90C26.

Key words and phrases. limited memory BFGS method, optimization, nonmonotone, global convergence.

This work is supported by China NSF grands 10761001 and the Scientific Research Foundation of Guangxi University (Grant No. X081082). 
where $g\left(x_{k}\right)=\nabla f\left(x_{k}\right)$ is the gradient of $f(x)$ at $x_{k}$, and $G\left(x_{k}\right)=\nabla^{2} f\left(x_{k}\right)$ is the Hessian matrix of $f(x)$ at current iteration. Moreover, the exact solution of the system (1.3) could be too burdensome, or is not necessary when $x_{k}$ is far from a solution [31]. Inexact Newton methods [8, 31] represent the basic approach underlying most of the Newton-type large-scale algorithms. At each iteration, the current estimate of the solution is updated by approximately solving the linear system (1.3) using an iterative algorithm. The inner iteration is typically "truncated" before the solution to the linear system is obtained. The limited memory BFGS (L-BFGS) method (see [3]) is an adaptation of the BFGS method for large-scale problems. The implementation is almost identical to that of the standard BFGS method, the only difference is that the inverse Hessian approximation is not formed explicitly, but defined by a small number of BFGS updates. It is often provided a fast rate of linear convergence, and requires minimal storage.

Since the standard BFGS is wildly used to solve general minimization problems, most of the studies concerning limited memory methods are concentrate on the L-BFGS method. We know that, the BFGS update exploits only the gradient information, while the information of function values available is neglected. Therefore, many efficient attempts have been made to modify the usual quasi-Newton methods using both the gradient and function values information (e.g. [41, 51]). Lately, in order to get a higher order accuracy in approximating the second curvature of the objective function, Wei, Li, and Qi [41], and Zhang, Deng, and Chen [51] proposed modified BFGS-type methods for (1.1), and the reported numerical results show that the average performance is better than that of the standard BFGS method, respectively.

The monotone line search technique is often used to get the stepsize $\alpha_{k}$, however monotonicity may cause a series of very small steps if the contours of objective function are a family of curves with large curvature [18]. More recently, the nonmonotonic line search for solving unconstrained optimization is proposed by Grippo et al. in [18]. Han and Liu [21] presented a new nonmonotone BFGS method for (1.1). The global convergence of the convex objective function was established. Numerical results show that this method is more competitive to the normal BFGS method with monotone line search. We [49] proved its superlinear convergence.

Motivated by the above observation, we propose two limited memory BFGStype method on the basic of Wei et al. [41], Zhang et al. [51], and [21], respectively, which are suitable for solving large-scale unconstrained optimization problems. The major contribution of this paper is an extension of the BFGS-type method in [41], [51], and the nonmonotone line search technique to limited memory scheme. The triple of the standard L-BFGS method $\left\{s_{i}, y_{i}\right\}$, $i=k-\widetilde{m}+1, \ldots, k$, is stored, where $s_{i}=x_{i+1}-x_{i}, y_{i}=g_{i+1}-g_{i}, g_{i}=g\left(x_{i}\right)$ and $g_{i+1}=g\left(x_{i+1}\right)$ are the gradient of $f(x)$ at $x_{i}$ and $x_{i+1}$, respectively, $\widetilde{m}>0$ is a constant. A distinguishing feature of our proposed L-BFGS method is that, 
at each iteration, a triple

$$
\left\{s_{i}, y_{i}, A_{i}\right\}, i=k-\widetilde{m}+1, \ldots, k,
$$

is stored, where $A_{i}$ is a scalar related to function value. Compared with the standard BFGS method, at each iteration, the proposed method requires no more function or derivative evaluations, and hardly more storage or arithmetic operations. Under suitable conditions, we establish the global convergence of the method. The numerical experiments of the proposed method on a set of large problems indicate that it is interesting.

This paper is organized as follows. In the next section, modified BFGS update and nonmonotone line search are stated. The proposed L-BFGS algorithms are given in Section 3. Under some reasonable conditions, the global convergence of the given methods is established in Section 4. Numerical results and a conclusion are presented in Section 5 and in Section 6, respectively.

\section{Modified BFGS update and nonmonotone line search}

Quasi-Newton methods are iterative methods of the form

$$
x_{k+1}=x_{k}+\alpha_{k} d_{k},
$$

where $x_{k}$ is the $k$ th iteration point, $\alpha_{k}$ is a stepsize, and $d_{k}$ is a search direction. Now we first state the search direction as follows.

\subsection{Some modified BFGS update formulas}

The search direction of the quasi-Newton method is defined by

$$
B_{k} d_{k}+g_{k}=0,
$$

where $g_{k}=g\left(x_{k}\right)=\nabla f\left(x_{k}\right)$ is the gradient of $f(x)$ at $x_{k}, B_{k}$ is an approximation of $\nabla^{2} f\left(x_{k}\right)$. By tradition, $\left\{B_{k}\right\}$ satisfies the following quasi-Newton equation

$$
B_{k+1} s_{k}=y_{k},
$$

where $s_{k}=x_{k+1}-x_{k}=\alpha_{k} d_{k}, y_{k}=g_{k+1}-g_{k}$. Throughout the paper, we use these notations: $\|\cdot\|$ is the Euclidean norm, $g\left(x_{k}\right)$ and $g\left(x_{k+1}\right)$ are replaced by $g_{k}$ and $g_{k+1}$, and $f\left(x_{k}\right)$ and $f\left(x_{k+1}\right)$ are replaced by $f_{k}$ and $f_{k+1}$ respectively. The famous update $B_{k}$ is the standard BFGS formula

$$
B_{k+1}=B_{k}-\frac{B_{k} s_{k} s_{k}^{T} B_{k}}{s_{k}^{T} B_{k} s_{k}}+\frac{y_{k} y_{k}^{T}}{y_{k}^{T} s_{k}}
$$

Let $H_{k}$ be the inverse of $B_{k}$. Then the inverse update formula of (2.3) method is represented as

$$
\begin{aligned}
H_{k+1} & =H_{k}-\frac{y_{k}^{T}\left(s_{k}-H_{k} y_{k}\right) s_{k} s_{k}^{T}}{\left(y_{k}^{T} s_{k}\right)^{2}}+\frac{\left(s_{k}-H_{k} y_{k}\right) s_{k}^{T}+s_{k}\left(s_{k}-H_{k} y_{k}\right)^{T}}{\left(y_{k}^{T} s_{k}\right)^{2}} \\
(2.4) & =\left(I-\frac{s_{k} y_{k}^{T}}{y_{k}^{T} s_{k}}\right) H_{k}\left(I-\frac{y_{k} s_{k}^{T}}{y_{k}^{T} s_{k}}\right)+\frac{s_{k} s_{k}^{T}}{y_{k}^{T} s_{k}},
\end{aligned}
$$


which is the dual form of the DFP update formula in the sense that $H_{k} \leftrightarrow B_{k}$, $H_{k+1} \leftrightarrow B_{k+1}$, and $s_{k} \leftrightarrow y_{k}$. It has been shown that the BFGS method is very efficient for solving unconstrained optimization problems (1.1) [11, 14, 47]. For convex minimization problems, the BFGS method are global convergence if the exact line search or some special inexact line search is used $[1,2,4$, $12,16,32,33,38]$ and its local convergence has been well established $[9,10$, 17]. For general function $f$, Dai [5] have constructed an example to show that the standard BFGS method may fail for non-convex functions with inexact line search, Mascarenhas [29] showed that the nonconvergence of the standard BFGS method even with exact line search.

In order to obtain a global convergence of BFGS method without convexity assumption on the objective function, Li and Fukushima [22, 23] made a slight modification to the standard BFGS method. Now we state their works as follows:

(i) A new quasi-Newton equation [22] with following form

$$
B_{k+1} s_{k}=y_{k}^{1 *}
$$

where $y_{k}^{1 *}=y_{k}+\left(\max \left\{0,-\frac{y_{k}^{T} s_{k}}{\left\|s_{k}\right\|^{2}}\right\}+\phi\left(\left\|g_{k}\right\|\right)\right) s_{k}$, and function $\phi: \mathbb{R} \rightarrow \mathbb{R}$ satisfies: (a) $\phi(t)>0$ for all $t>0$; (b) $\phi(t)=0$ if and only if $t=0$; (c) $\phi(t)$ is bounded if $t$ is in a bounded set.

(ii) A modified BFGS update formula [23] with following form

$$
B_{k+1}= \begin{cases}B_{k}-\frac{B_{k} s_{k} s_{k}^{T} B_{k}}{s_{k}^{T} B_{k} s_{k}}+\frac{y_{k}^{1 *} y_{k}^{1 * T}}{y_{k}^{1 * T} s_{k}}, & \text { if } \frac{s_{k}^{T} y_{k}^{1 *}}{\left\|s_{k}\right\|^{2}} \geq \phi\left(\left\|g_{k}\right\|\right) \\ B_{k}, & \text { otherwise. }\end{cases}
$$

Then it is not difficult to see that $s_{k}^{T} y_{k}^{1 *}>0$ always holds, which can ensure that the update matrix $B_{k+1}$ inherits the positive definiteness of $B_{k}$ (see [14]). The global convergence and the superlinear convergence of these two methods for nonconvex have been established under appropriate conditions (see [22, 23] in detail).

In order to get a better approximation of the objective function Hessian matrix, Wei, Li, and Qi [41] and Zhang, Deng, and Chen [51] proposed modified quasi-Newtion equations which are given as follows. (i) The equation of Wei, Li, and Qi [41]:

$$
B_{k+1} s_{k}=y_{k}^{2 *}=y_{k}+A_{k} s_{k},
$$

where

$$
A_{k}=\frac{2\left[f\left(x_{k}\right)-f\left(x_{k+1}\right)\right]+\left[g\left(x_{k+1}\right)+g\left(x_{k}\right)\right]^{T} s_{k}}{\left\|s_{k}\right\|^{2}} .
$$

They replaced all the $y_{k}$ in (2.3), and obtained the following modified BFGStype update formula

$$
B_{k+1}=B_{k}-\frac{B_{k} s_{k} s_{k}^{T} B_{k}}{s_{k}^{T} B_{k} s_{k}}+\frac{y_{k}^{2 *} y_{k}^{2 *^{T}}}{y_{k}^{2 *^{T}} s_{k}} .
$$


Note that this quasi-Newton equation (2.6) contains both gradient and function value information at the current and the previous step, one may argue that the resulting methods will really outperform than the original method. In fact, the practical computation shows that this method is better than the normal BFGS method (see [41, 42] for detail) for some given problems [30]. Furthermore, some theoretical advantages of the new quasi-Newton equation (2.6) can be seen from the following two theorems.

Theorem 2.1 ([42, Lemma 3.1]). Considering the quasi-Newton equation (2.6). Then we have for all $k \geq 1$

$$
f\left(x_{k}\right)=f\left(x_{k+1}\right)+g\left(x_{k+1}\right)^{T}\left(x_{k}-x_{k+1}\right)+\frac{1}{2}\left(x_{k}-x_{k+1}\right)^{T} B_{k+1}\left(x_{k}-x_{k+1}\right) .
$$

Theorem 2.2 ([24, Theorem 3.1]). Assume that the function $f(x)$ is sufficiently smooth and $\left\|s_{k}\right\|$ is sufficiently small. Then we have

$$
s_{k}^{T} G_{k+1} s_{k}-s_{k}^{T} y_{k}^{2 *}-\frac{1}{3} s_{k}^{T}\left(T_{k+1} s_{k}\right) s_{k}=O\left(\left\|s_{k}\right\|^{4}\right)
$$

and

$$
s_{k}^{T} G_{k+1} s_{k}-s_{k}^{T} y_{k}-\frac{1}{2} s_{k}^{T}\left(T_{k+1} s_{k}\right) s_{k}=O\left(\left\|s_{k}\right\|^{4}\right),
$$

where $G_{k+1}$ denotes the Hessian matrix of $f$ at $x_{k+1}, T_{k+1}$ is the tensor of $f$ at $x_{k+1}$, and

$$
s_{k}^{T}\left(T_{k+1} s_{k}\right) s_{k}=\sum_{i, j, l=1}^{n} \frac{\partial^{3} f\left(x_{k+1}\right)}{\partial x^{i} \partial x^{j} \partial x^{l}} s_{k}^{i} s_{k}^{j} s_{k}^{l} .
$$

(iii) The equation of Zhang, Deng, and Chen [51]:

$$
B_{k+1} s_{k}=y_{k}^{3 *}=y_{k}+\bar{A}_{k} s_{k},
$$

where

$$
\bar{A}_{k}=\frac{6\left[f\left(x_{k}\right)-f\left(x_{k+1}\right)\right]+3\left[g\left(x_{k+1}\right)+g\left(x_{k}\right)\right]^{T} s_{k}}{\left\|s_{k}\right\|^{2}} .
$$

They replaced all the $y_{k}$ in (2.3), and obtained the following modified BFGStype update formula

$$
B_{k+1}=B_{k}-\frac{B_{k} s_{k} s_{k}^{T} B_{k}}{s_{k}^{T} B_{k} s_{k}}+\frac{y_{k}^{3 *} y_{k}^{3 *^{T}}}{y_{k}^{3 *^{T}} s_{k}} .
$$

Similar to equation (2.6), this quasi-Newton equation (2.11) contains both gradient and function value information at the current and the previous step, one may argue that the resulting methods will really outperform than the original method. In fact, the practical computation shows that this method is better than the normal BFGS method (see [51] for detail). Furthermore, some theoretical advantages of the new quasi-Newton equation (2.11) can be seen from the following theorem. 
Theorem 2.3 ([51, Theorem 3.3]). Assume that the function $f(x)$ is sufficiently smooth and $\left\|s_{k}\right\|$ is sufficiently small. Then we have

$$
s_{k}^{T}\left(G_{k+1} s_{k}-y_{k}^{3 *}\right)=O\left(\left\|s_{k}\right\|^{4}\right)
$$

and

$$
s_{k}^{T}\left(G_{k+1} s_{k}-y_{k}\right)=O\left(\left\|s_{k}\right\|^{3}\right) .
$$

It is not difficult to deduce that $s_{k}^{T} y_{k}^{2 *}>0$ holds for the uniformly convex function $f$ (or see [42]). We all know that the condition $s_{k}^{T} y_{k}^{2 *}>0$ can ensure that the update matrix $B_{k+1}$ from (2.8) inherits the positive definiteness of $B_{k}$. Similarly, in order to get the positive definiteness of $B_{k}$ in (2.13) for each $k$, we give a modified BFGS update of (2.13), i.e., the modified update formula is defined by

$$
B_{k+1}=B_{k}-\frac{B_{k} s_{k} s_{k}^{T} B_{k}}{s_{k}^{T} B_{k} s_{k}}+\frac{y_{k}^{4 *} y_{k}^{4{ }^{T}}}{y_{k}^{4 *^{T}} s_{k}},
$$

where $y_{k}^{4 *}=y_{k}+A_{k}^{*} s_{k}, A_{k}^{*}=\frac{1}{3} \max \left\{\bar{A}_{k}, 0\right\}$. Then the corresponding quasiNewton equation is

$$
B_{k+1} s_{k}=y_{k}^{4 *} \text {. }
$$

From the definition of $y_{k}^{4 *}$, we can obtain $s_{k}^{T} y_{k}^{4 *}>0$ if the objective function $f$ is uniformly convex function (see Lemma 4.1).

There are other modified formulas that can be seen from [7, 34] in detail, here we do not present anymore.

\subsection{One nonmonotone line search}

Normally the steplength $\alpha_{k}$ is generated by the following weak Wolfe-Powell (WWP): Find a steplength $\alpha_{k}$ such that

$$
\begin{gathered}
f\left(x_{k}+\alpha_{k} d_{k}\right) \leq f\left(x_{k}\right)+\sigma_{1} \alpha_{k} g_{k}^{T} d_{k}, \\
g\left(x_{k}+\alpha_{k} d_{k}\right)^{T} d_{k} \geq \sigma_{2} g_{k}^{T} d_{k},
\end{gathered}
$$

where $0<\sigma_{1}<\sigma_{2}<1$. Many authors analysis the BFGS algorithm from generalizing line search procedures $[25,26]$. Recently, the nonmonotone line search technique for unconstrained optimization is proposed by Grippo et. al. $[18,19,20]$ and further studied by [27, 28] etc.. Grippo, Lamparillo, and Lucidi [18] proposed the following nonmonotone line search we call GLL line search.

GLL line search: Select steplength $\alpha_{k}$ satisfying

$$
\begin{gathered}
f\left(x_{k+1}\right) \leq \max _{0 \leq j \leq n(k)} f\left(x_{k-j}\right)+\varepsilon_{1} \alpha_{k} g_{k}^{T} d_{k}, \\
g\left(x_{k+1}\right)^{T} d_{k} \geq \max \left\{\varepsilon_{2}, 1-\left(\alpha_{k}\left\|d_{k}\right\|\right)^{p}\right\} g_{k}^{T} d_{k},
\end{gathered}
$$

where $p \in(-\infty, 1), k=0,1,2, \ldots, \varepsilon_{1}, \varepsilon_{2} \in(0,1), n(k)=\min \{H, k\}, H \geq 0$ is an integer constant. Combining this line search and the normal BFGS formula (2.3), Han and Liu [21] established the global convergence of the convex 
objective function. Numerical results show that this method is more competitive than the normal BFGS method with WWP line search. Recently, the superlinear convergence of the new nonmonotone BFGS algorithm for convex function was proved by Yuan and Wei [49].

\section{Limited memory BFGS-type method}

In this section, we propose new algorithm to solve (1.1). To improve the performance of the line search, it is a better choice to use the GLL line search instead of the WWP line search. Then our method generates a sequence of points $\left\{x_{k}\right\}$ by

$$
x_{k+1}=x_{k}+\alpha_{k} d_{k}, k=0,1,2, \ldots,
$$

where $\alpha_{k}$ is determined by (2.20) and (2.21), $d_{k}$ is a descent direction of $f$ at $x_{k}$. In the following, we state the direction $d_{k}$ in details.

The limited memory BFGS (L-BFGS) method (see [3]) is an adaptation of the BFGS method for large-scale problems. In the L-BFGS method, matrix $H_{k}$ is obtained by updating the basic matrix $H_{0} \widetilde{m}(>0)$ times using BFGS formula with the previous $\widetilde{m}$ iterations. The standard BFGS correction (2.4) has the following form

$$
H_{k+1}=V_{k}^{T} H_{k} V_{k}+\rho_{k} s_{k} s_{k}^{T}
$$

where $\rho_{k}=\frac{1}{s_{k}^{T} y_{k}}, V_{k}=I-\rho_{k} y_{k} s_{k}^{T}, I$ is the unit matrix. Thus, $H_{k+1}$ in the L-BFGS method has the following form:

$$
\begin{aligned}
H_{k+1}= & V_{k}^{T} H_{k} V_{k}+\rho_{k} s_{k} s_{k}^{T} \\
= & V_{k}^{T}\left[V_{k-1}^{T} H_{k-1} V_{k-1}+\rho_{k-1} s_{k-1} s_{k-1}^{T}\right] V_{k}+\rho_{k} s_{k} s_{k}^{T} \\
= & \cdots \\
= & {\left[V_{k}^{T} \cdots V_{k-\widetilde{m}+1}^{T}\right] H_{k-\widetilde{m}+1}\left[V_{k-\widetilde{m}+1} \cdots V_{k}\right] } \\
& +\rho_{k-\widetilde{m}+1}\left[V_{k-1}^{T} \cdots V_{k-\widetilde{m}+2}^{T}\right] s_{k-\widetilde{m}+1} s_{k-\widetilde{m}+1}^{T}\left[V_{k-\widetilde{m}+2} \cdots V_{k-1}\right] \\
& +\cdots \\
& +\rho_{k} s_{k} s_{k}^{T} .
\end{aligned}
$$

To improve the performance of the standard limited memory BFGS algorithm, it is a better choice use the modified BFGS-type update instead of the standard BFGS. If we replaced all the $y_{k}$ with $y_{k}^{4 *}$ and $y_{k}^{2 *}$ in (3.2) respectively, the new limited memory BFGS-type update can be obtained by

$$
\begin{aligned}
H_{k+1}= & V_{k}^{* T} H_{k} V_{k}^{*}+\rho_{k}^{*} s_{k} s_{k}^{T} \\
= & V_{k}^{* T}\left[V_{k-1}^{*}{ }^{T} H_{k-1} V_{k-1}^{*}+\rho_{k-1}^{*} s_{k-1} s_{k-1}^{T}\right] V_{k}^{*}+\rho_{k}^{*} s_{k} s_{k}^{T} \\
= & \cdots \\
= & {\left[V_{k}^{* T} \cdots V_{k-\widetilde{m}+1}^{T}\right] H_{k-\widetilde{m}+1}\left[V_{k-\widetilde{m}+1}^{*} \cdots V_{k}^{*}\right] } \\
& +\rho_{k-\widetilde{m}+1}^{*}\left[V_{k-1}^{*} \cdots V_{k-\widetilde{m}+2}^{T}\right] s_{k-\widetilde{m}+1} s_{k-\widetilde{m}+1}^{T}\left[V_{k-\widetilde{m}+2}^{*} \cdots V_{k-1}^{*}\right] \\
& +\cdots
\end{aligned}
$$




$$
+\rho_{k}^{*} s_{k} s_{k}^{T}
$$

and

$$
\begin{aligned}
H_{k+1}= & V_{k}^{* * T} H_{k} V_{k}^{* *}+\bar{\rho}_{k}^{*} s_{k} s_{k}^{T} \\
= & V_{k}^{* * T}\left[V_{k-1}^{* * T} H_{k-1} V_{k-1}^{* *}+\bar{\rho}_{k-1}^{*} s_{k-1} s_{k-1}^{T}\right] \bar{V}_{k}^{* *}+\bar{\rho}_{k}^{*} s_{k} s_{k}^{T} \\
= & \cdots \\
= & {\left[V_{k}^{* * T} \cdots V_{k-\widetilde{m}+1}^{* *}\right] H_{k-\widetilde{m}+1}\left[V_{k-\widetilde{m}+1}^{* *} \cdots \bar{V}_{k}^{* *}\right] } \\
& +\bar{\rho}_{k-\widetilde{m}+1}^{*}\left[V_{k-1}^{* *} T \cdots V_{k-\widetilde{m}+2}^{* *}\right] s_{k-\widetilde{m}+1} s_{k-\widetilde{m}+1}^{T}\left[V_{k-\widetilde{m}+2}^{* *} \cdots V_{k-1}^{* *}\right] \\
& +\cdots \\
& +\bar{\rho}_{k}^{*} s_{k} s_{k}^{T},
\end{aligned}
$$

where $\rho_{k}^{*}=\frac{1}{s_{k}^{T} y_{k}^{4 *}}, V_{k}^{*}=I-\rho_{k}^{*} y_{k}^{4 *} s_{k}^{T}$, and $\bar{\rho}_{k}^{*}=\frac{1}{s_{k}^{T} y_{k}^{2 *}}, V_{k}^{* *}=I-\bar{\rho}_{k}^{*} y_{k}^{2 *} s_{k}^{T}$.

Now we state the new limited memory BFGS-type algorithm (L-BFGS-A) with GLL line search as follows.

\section{Algorithm 1. (L-BFGS-A1)}

Step 0: Choose an initial point $x_{0} \in R^{n}$, an basic symmetric positive definite matrix $H_{0} \in R^{n \times n}$, and constants $r, \varepsilon_{1}, \varepsilon_{2} \in(0,1), p \in(-\infty, 1), H \geq 0$, an positive integer $m_{1}$. Let $k:=0$;

Step 1: Stop if $\left\|g_{k}\right\|=0$.

Step 2: Determine $d_{k}$ by

$$
d_{k}=-H_{k} g_{k} .
$$

Step 3: Find $\alpha_{k}$ satisfying (2.20) and (2.21).

Step 4: Let the next iterative be $x_{k+1}=x_{k}+\alpha_{k} d_{k}$.

Step 5: Let $\widetilde{m}=\min \left\{k+1, m_{1}\right\}$. Update $H_{0}$ for $\widetilde{m}$ times to get $H_{k+1}$ by (3.3).

Step 6: Let $k:=k+1$. Go to step 1 .

\section{Algorithm 11. (L-BFGS-A11)}

Step 5: Let $\widetilde{m}=\min \left\{k+1, m_{1}\right\}$. Update $H_{0}$ for $\widetilde{m}$ times to get $H_{k+1}$ by (3.4).

In the following, we assume that the algorithm updates $B_{k}$-the inverse of $H_{k}$. We also assume that the basic matrix $B_{0}$, and its inverse $H_{0}$, are bounded and positive definite. The Algorithm 1 with $B_{k}$ can be stated as follows.

Algorithm 2. (L-BFGS-A2)

Step 2: Determine $d_{k}$ by

$$
B_{k} d_{k}=-g_{k} .
$$

Step 5: Let $\widetilde{m}=\min \left\{k+1, m_{1}\right\}$. Put $s_{k}=x_{k+1}-x_{k}=\alpha_{k} d_{k}, y_{k}=g_{k+1}-g_{k}$. Update $B_{0}$ for $\widetilde{m}$ times, i.e., for $l=k-\widetilde{m}+1, \ldots, k$ compute

$$
B_{k}^{l+1}=B_{k}^{l}-\frac{B_{k}^{l} s_{l} s_{l}^{T} B_{k}^{l}}{s_{l}^{T} B_{k}^{l} s_{l}}+\frac{y_{l}^{4 *} y_{l}^{4 * T}}{y_{l}^{4 * T} s_{l}}
$$

where $s_{l}=x_{l+1}-x_{l}, y_{l}^{4 *}=y_{l}+A_{l}^{*} s_{l}$, and $B_{k}^{k-\widetilde{m}+1}=B_{0}$ for all $k$. 
Algorithm 22. (L-BFGS-A22)

Step 2: Determine $d_{k}$ by

$$
B_{k} d_{k}=-g_{k} .
$$

Step 5: Let $\widetilde{m}=\min \left\{k+1, m_{1}\right\}$. Put $s_{k}=x_{k+1}-x_{k}=\alpha_{k} d_{k}, y_{k}=g_{k+1}-g_{k}$. Update $B_{0}$ for $\widetilde{m}$ times, i.e., for $l=k-\widetilde{m}+1, \ldots, k$ compute

$$
B_{k}^{l+1}=B_{k}^{l}-\frac{B_{k}^{l} s_{l} s_{l}^{T} B_{k}^{l}}{s_{l}^{T} B_{k}^{l} s_{l}}+\frac{y_{l}^{2 *} y_{l}^{2 * T}}{y_{l}^{2 * T} s_{l}}
$$

where $s_{l}=x_{l+1}-x_{l}, y_{l}^{2 *}=y_{l}+A_{l} s_{l}$, and $B_{k}^{k-\widetilde{m}+1}=B_{0}$ for all $k$.

Note that Algorithms 1 and 2 are mathematically equivalent, and Algorithms 11 and 22 are mathematically equivalent too. In our numerical experiments we implement Algorithms 1 and 11, and Algorithms 2 and 22 are given only for the purpose of analysis. Throughout this paper, we only discuss Algorithms 2 and 22. In the following section, we will concentrate on their global convergence.

\section{Convergence analysis}

This section is devoted to show that Algorithm 2 is convergent on twice continuously differentiable and uniformly convex function. In order to establish global convergence for Algorithm 2, we need the following assumptions.

Assumption A. (i) The level set $\Omega=\left\{x \mid f(x) \leq f\left(x_{0}\right)\right\}$ is bounded.

(ii) The function $f$ is twice continuously differentiable on $\Omega$.

(iii) The function $f$ is uniformly convex, i.e., there exist positive constants $m$ and $M$ such that

$$
m\|d\|^{2} \leq d^{T} G(x) d \leq M\|d\|^{2}
$$

holds for all $x \in \Omega$ and $d \in \mathbb{R}^{n}$, where $G(x)=\nabla^{2} f(x)$. These assumptions are the same as those in $[42,51]$.

It is obvious that Assumption A implies that there exists a constant $M_{*}>0$ such that

$$
\|G(x)\| \leq M_{*}, x \in \Omega .
$$

Assumption A (ii) implies that there exists a constant $L \geq 0$ satisfying

$$
\|g(x)-g(y)\| \leq L\|x-y\|, x, y \in \Omega .
$$

Lemma 4.1. Let Assumption A hold. Then there exists a positive number $M_{1}$ such that

$$
\frac{\left\|y_{k}^{4 *}\right\|^{2}}{s_{k}^{T} y_{k}^{4 *}} \leq M_{1}, k=0,1,2, \ldots
$$

Proof. Following the definition of $y_{k}^{4 *}$ and the Taylor's formula, we get

$$
\begin{aligned}
s_{k}^{T} y_{k}^{4 *} & =s_{k}^{T} y_{k}+s_{k}^{T} A_{k}^{*} s_{k} \\
& =\max \left\{2\left[f_{k}-f_{k+1}\right]+2 g_{k+1}^{T} s_{k}, s_{k}^{T} y_{k}\right\}
\end{aligned}
$$




$$
\begin{gathered}
=\max \left\{2 \left[-g_{k+1}^{T} s_{k}+\frac{1}{2} s_{k}^{T} G\left(x_{k}+\theta\left(x_{k+1}-x_{k}\right) s_{k}\right]+2 g_{k+1}^{T} s_{k},\right.\right. \\
s_{k}^{T} G\left(x_{k}+\theta_{1}\left(x_{k+1}-x_{k}\right) s_{k}\right\} \\
=\max \left\{s _ { k } ^ { T } G \left(x_{k}+\theta\left(x_{k+1}-x_{k}\right) s_{k}, s_{k}^{T} G\left(x_{k}+\theta_{1}\left(x_{k+1}-x_{k}\right) s_{k}\right\},\right.\right.
\end{gathered}
$$

where $\theta, \theta_{1} \in(0,1)$. Combining with Assumption A(iii), it is easy to obtain

$$
m\left\|s_{k}\right\|^{2} \leq s_{k}^{T} y_{k}^{4 *} \leq M\left\|s_{k}\right\| .
$$

By the definition of $y_{k}^{4 *}$ and the Taylor's formula again, we have

$$
\begin{aligned}
\left\|y_{k}^{4 *}\right\| & =\left\|y_{k}+\max \left\{\frac{2\left[f\left(x_{k}\right)-f\left(x_{k+1}\right)\right]+\left[g\left(x_{k+1}\right)+g\left(x_{k}\right)\right]^{T} s_{k}}{\left\|s_{k}\right\|^{2}}, 0\right\} s_{k}\right\| \\
& \leq \max \left\{\left\|y_{k}\right\|+\frac{\left|2\left[f\left(x_{k}\right)-f\left(x_{k+1}\right)\right]+\left[g\left(x_{k+1}\right)+g\left(x_{k}\right)\right]^{T} s_{k}\right|}{\left\|s_{k}\right\|},\left\|y_{k}\right\|\right\} \\
& \leq 2\left\|y_{k}\right\|+\frac{\mid s_{k}^{T} G\left(x_{k}+\theta\left(x_{k+1}-x_{k}\right) s_{k} \mid\right.}{\left\|s_{k}\right\|^{2}} \\
& \leq 2 L\left\|s_{k}\right\|+M\left\|s_{k}\right\| \\
(4.4) & =(2 L+M)\left\|s_{k}\right\|,
\end{aligned}
$$

where $\theta \in(0,1)$, the third inequality follows (4.1) and (4.2). By (4.3) and (4.4), we get

$$
\frac{\left\|y_{k}^{4 *}\right\|^{2}}{s_{k}^{T} y_{k}^{4 *}} \leq \frac{(2 L+M)^{2}\left\|s_{k}\right\|^{2}}{m\left\|s_{k}\right\|^{2}}=\frac{(2 L+M)^{2}}{m}=M_{1} .
$$

The proof is complete.

Lemma 4.2. Let $B_{k}$ be generated by (3.7). Then we have

$$
\operatorname{det}\left(B_{k+1}\right)=\operatorname{det}\left(B_{k}^{k-\widetilde{m}+1}\right) \prod_{l=k-\widetilde{m}+1}^{k} \frac{s_{l}^{T} y_{l}^{4 *}}{s_{l}^{T} B_{l} s_{l}},
$$

where $\operatorname{det}\left(B_{k}\right)$ denotes the determinant of $B_{k}$.

Proof. To begin with, we take the determinant in both sides of (2.8)

$$
\begin{aligned}
& \operatorname{det}\left(B_{k+1}\right)=\operatorname{det}\left(B_{k}\left(I-\frac{s_{k} s_{k}^{T} B_{k}}{s_{k}^{T} B_{k} s_{k}}+\frac{B_{k}^{-1} y_{k}^{4 *} y_{k}^{4 * T}}{s_{k}^{T} y_{k}^{4 *}}\right)\right) \\
&=\operatorname{det}\left(B_{k}\right) \operatorname{det}\left(I-\frac{s_{k} s_{k}^{T} B_{k}}{s_{k}^{T} B_{k} s_{k}}+\frac{B_{k}^{-1} y_{k}^{4 *} y_{k}^{4 * T}}{s_{k}^{T} y_{k}^{4 *}}\right) \\
&=\operatorname{det}\left(B_{k}\right)(\left(1-s_{k}^{T} \frac{B_{k} s_{k}}{s_{k}^{T} B_{k} s_{k}}\right)\left(1+\left(B_{k}^{-1} y_{k}^{4 *}\right)^{T} \frac{y_{k}^{4 *}}{y_{k}^{4 * T} s_{k}}\right) \\
&\left.-\left(-s_{k}^{T} \frac{y_{k}^{4 *}}{y_{k}^{4 * T} s_{k}}\right)\left(\frac{\left(B_{k} s_{k}\right)^{T}}{s_{k}^{T} B_{k} s_{k}} B_{k}^{-1} y_{k}^{4 *}\right)\right)
\end{aligned}
$$




$$
=\operatorname{det}\left(B_{k}\right) \frac{y_{k}^{4 * T} s_{k}}{s_{k}^{T} B_{k} s_{k}}
$$

where the third equality follows from the formula (see, e.g., [9, Lemma 7.6])

$$
\operatorname{det}\left(I+u_{1} u_{2}^{T}+u_{3} u_{4}^{T}\right)=\left(1+u_{1}^{T} u_{2}\right)\left(1+u_{3}^{T} u_{4}\right)-\left(u_{1}^{T} u_{4}\right)\left(u_{2}^{T} u_{3}\right) .
$$

Therefore, there is also a simple expression for the determinant of (3.7)

$$
\operatorname{det}\left(B_{k+1}\right)=\operatorname{det}\left(B_{k}^{k-\widetilde{m}+1}\right) \prod_{l=k-\widetilde{m}+1}^{k} \frac{s_{l}^{T} y_{l}^{4 *}}{s_{l}^{T} B_{l} s_{l}} .
$$

Then we complete the proof.

Define the length of the orthogonal projection of $-g_{k}$ on $d_{k}$ by

$$
\eta_{k}=\frac{-g_{k}^{T} d_{k}}{\left\|d_{k}\right\|} .
$$

The following Lemmas 4.3-4.6 has been proved in [21], here we only state them as follows, but omit the proof.

Lemma 4.3. Let Assumption A be satisfied. Consider GLL line search. Then there exists a positive constant $b_{0}$ such that

$$
\left\|s_{k}\right\| \geq b_{0} \min \left\{\eta_{k},\left(\eta_{k}\right)^{\frac{1}{1-p}}\right\}
$$

where $\eta_{k}$ is defined by (4.6).

Lemma 4.4. Denote that

$$
f\left(x_{l(k)}\right)=\max _{0 \leq j \leq n(k)} f\left(x_{k-j}\right), k-n(k) \leq l(k) \leq k .
$$

If $f_{k+1} \leq f\left(x_{l(k)}\right), k=0,1,2, \ldots$, then the sequence $\left\{f\left(x_{l(k)}\right)\right\}$ monotonically decreases, and $x_{k} \in \Omega$ for all $k \geq 0$.

Lemma 4.5. If

$$
f_{k+1} \leq f\left(x_{l(k)}\right)-t_{k}, k=0,1,2, \ldots,
$$

where $t_{k} \geq 0$, then

$$
\sum_{k=0}^{\infty} \min _{0 \leq j \leq n(k)} t_{k+n(k)-j}<+\infty
$$

Lemma 4.6. If the sequence of nonnegative numbers $m_{k}(k=0,1, \ldots)$ satisfies

$$
\prod_{j=0}^{k} m_{j} \geq c_{1}^{k}, c_{1}>0, k=1,2, \ldots,
$$

then $\lim \sup _{k} m_{k}>0$. 
Lemma 4.7. Let $\left\{x_{k}\right\}$ be generated by Algorithm 2 and Assumption A hold. If

$$
\liminf _{k \rightarrow \infty}\left\|g_{k}\right\|>0
$$

then there exists a constant $\epsilon_{0}>0$ such that

$$
\prod_{j=0}^{k} \eta_{j} \geq\left(\epsilon_{0}\right)^{k+1} \quad \text { for all } k \geq 0 \text {. }
$$

Proof. Assume that $\liminf _{k}\left\|g_{k}\right\|>0$, i.e., there exists a constant $c_{2}>0$ such that

$$
\left\|g_{k}\right\| \geq c_{2}, k=0,1,2, \ldots
$$

From Assumption A(iii) and Taylor's formula, we have

$$
m\left\|s_{k}\right\|^{2} \leq s_{k}^{T} G\left(x_{k}+\theta_{1} s_{k}\right) s_{k}=s_{k}^{T} y_{k} \leq M\left\|s_{k}\right\|^{2},
$$

combining with (4.3), we get

$$
\frac{m}{M} s_{k}^{T} y_{k} \leq s_{k}^{T} y_{k}^{4 *} \leq \frac{M}{m} s_{k}^{T} y_{k} .
$$

Taking the trace operation in both sides of (3.7), we get

$$
\operatorname{Tr}\left(B_{k+1}\right)=\operatorname{Tr}\left(B_{k}^{k-\widetilde{m}+1}\right)-\sum_{l=k-\widetilde{m}+1}^{k} \frac{\left\|B_{l} s_{l}\right\|^{2}}{s_{l}^{T} B_{l} s_{l}}+\sum_{l=k-\widetilde{m}+1}^{k} \frac{\left\|y_{l}^{4 *}\right\|^{2}}{s_{l}^{T} y_{l}^{4 *}},
$$

where $\operatorname{Tr}\left(B_{k}\right)$ denotes the trace of $B_{k}$. Repeating this trace operation, we have

$$
\begin{aligned}
\operatorname{Tr}\left(B_{k+1}\right) & =\operatorname{Tr}\left(B_{k}^{k-\widetilde{m}+1}\right)-\sum_{l=k-\widetilde{m}+1}^{k} \frac{\left\|B_{l} s_{l}\right\|^{2}}{s_{l}^{T} B_{l} s_{l}}+\sum_{l=k-\widetilde{m}+1}^{k} \frac{\left\|y_{l}^{4 *}\right\|^{2}}{s_{l}^{T} y_{l}^{4 *}} \\
& =\cdots \\
& =\operatorname{Tr}\left(B_{0}\right)-\sum_{l=0}^{k} \frac{\left\|B_{l} s_{l}\right\|^{2}}{s_{l}^{T} B_{l} s_{l}}+\sum_{l=0}^{k} \frac{\left\|y_{l}^{4 *}\right\|^{2}}{s_{l}^{T} y_{l}^{4 *}} .
\end{aligned}
$$

Combining (4.10), (4.14), (3.5), (3.8), and Lemma 4.1, we obtain

$$
\operatorname{Tr}\left(B_{k+1}\right) \leq \operatorname{Tr}\left(B_{0}\right)-\sum_{j=0}^{k} \frac{c_{2}^{2}}{g_{j}^{T} H_{j} g_{j}}+(k+1) M_{1} .
$$

Using $B_{k+1}$ is positive definite, we have $\operatorname{Tr}\left(B_{k+1}\right)>0$. By (4.15), we obtain

$$
\sum_{j=0}^{k} \frac{c_{2}^{2}}{g_{j}^{T} H_{j} g_{j}} \leq \frac{\operatorname{Tr}\left(B_{0}\right)+(k+1) M_{1}}{c_{2}^{2}}
$$

and

$$
\operatorname{Tr}\left(B_{k+1}\right) \leq \operatorname{Tr}\left(B_{0}\right)+(k+1) M_{1}
$$


By the geometric-arithmetic mean value formula we get

$$
\prod_{j=0}^{k} g_{j}^{T} H_{j} g_{j} \geq\left[\frac{(k+1) c_{2}^{2}}{\operatorname{Tr}\left(B_{0}\right)+(k+1) M_{1}}\right]^{k+1} .
$$

Using Lemma 4.2, (4.12), and (2.21), we have

$$
\begin{aligned}
\operatorname{det}\left(B_{k+1}\right) & =\operatorname{det}\left(B_{k}^{k-\widetilde{m}+1}\right) \prod_{l=k-\widetilde{m}+1}^{k} \frac{s_{l}^{T} y_{l}^{4 *}}{s_{l}^{T} B_{l} s_{l}} \\
& \geq \operatorname{det}\left(B_{k}^{k-\widetilde{m}+1}\right) \prod_{l=k-\widetilde{m}+1}^{k} \frac{m}{M} \frac{s_{l}^{T} y_{l}}{s_{l}^{T} B_{l} s_{l}} \\
& \geq \operatorname{det}\left(B_{k}^{k-\widetilde{m}+1}\right) \prod_{l=k-\widetilde{m}+1}^{k} \frac{m}{M} \frac{\min \left\{1-\varepsilon_{2},\left\|s_{l}\right\|^{p}\right\}}{\alpha_{l}} \\
& \geq \cdots \operatorname{det}\left(B_{0}\right)\left[\frac{m}{M}\right]^{k+1} \prod_{j=0}^{k} \frac{\min \left\{1-\varepsilon_{2},\left\|s_{j}\right\|^{p}\right\}}{\alpha_{j}},
\end{aligned}
$$

which implies

$$
\frac{\operatorname{det}\left(B_{0}\right)}{\operatorname{det}\left(B_{k+1}\right)} \leq\left[\frac{m}{M}\right]^{k+1} \prod_{j=0}^{k} \max \left\{\frac{\alpha_{j}}{1-\varepsilon_{2}}, \frac{\alpha_{j}}{\left\|s_{j}\right\|^{p}}\right\} .
$$

By using the geometric-arithmetic mean value formula again, we get

$$
\operatorname{det}\left(B_{k+1}\right) \leq\left[\frac{\operatorname{Tr}\left(B_{k+1}\right)}{n}\right]^{n} .
$$

Using (4.17), (4.19) and (4.20), we obtain

$$
\begin{aligned}
\prod_{j=0}^{k} \max \left\{\frac{\alpha_{j}}{1-\varepsilon_{2}}, \frac{\alpha_{j}}{\left\|s_{j}\right\|^{p}}\right\} & \geq\left[\frac{m}{M}\right]^{k+1} \frac{\operatorname{det}\left(B_{0}\right) n^{n}}{\left[\operatorname{Tr}\left(B_{0}\right)+(k+1) M_{1}\right]^{n}} \\
& \geq\left[\frac{m}{M}\right]^{k+1} \frac{1}{k+1} \frac{\operatorname{det}\left(B_{0}\right) n^{n}}{\left[\operatorname{Tr}\left(B_{0}\right)+M_{1}\right]^{n}} \\
& \geq\left[\frac{m}{M}\right]^{k+1}\left(\frac{1}{\exp (n)}\right)^{k+1} \min \left\{\frac{\operatorname{det}\left(B_{0}\right) n^{n}}{\left[\operatorname{Tr}\left(B_{0}\right)+M_{1}\right]^{n}}, 1\right\} \\
& \geq\left[\frac{M}{\exp (n) m}\right]^{k+1} \min \left\{\frac{\operatorname{det}\left(B_{0}\right) n^{n}}{\left[\operatorname{Tr}\left(B_{0}\right)+M_{1}\right]^{n}}, 1\right\} \\
& \geq c_{3}^{k+1},
\end{aligned}
$$

where $c_{3} \leq\left[\frac{M}{\exp (n) m}\right] \min \left\{\frac{\operatorname{det}\left(B_{0}\right) n^{n}}{\left[\operatorname{Tr}\left(B_{0}\right)+M_{1}\right]^{n}}, 1\right\}$. Let

$$
\cos \theta_{j}=\frac{-g_{j}^{T} d_{j}}{\left\|g_{j}\right\|\left\|d_{j}\right\|}
$$


Multiplying (4.18) with (4.21), we get for all $k \geq 0$

$$
\begin{aligned}
\prod_{j=0}^{k} \max \left\{\frac{\left\|s_{j}\right\|\left\|g_{j}\right\| \cos \theta_{j}}{1-\varepsilon_{2}}, \frac{\left\|g_{j}\right\| \cos \theta_{j}}{\left.\left\|s_{j}\right\|^{p-1}\right\}}\right. & \geq c_{3}^{k+1}\left[\frac{(k+1) c_{2}^{2}}{\operatorname{Tr}\left(B_{0}\right)+(k+1) M_{1}}\right]^{k+1} \\
& \geq\left[\frac{c_{3} c_{2}^{2}}{\operatorname{Tr}\left(B_{0}\right)+M_{1}}\right]^{k+1} .
\end{aligned}
$$

By

$$
\begin{aligned}
& \prod_{j=0}^{k} \max \left\{\frac{\left\|s_{j}\right\|\left\|g_{j}\right\| \cos \theta_{j}}{1-\varepsilon_{2}}, \frac{\left\|g_{j}\right\| \cos \theta_{j}}{\left\|s_{j}\right\|^{p-1}}\right\} \\
\leq & \left(\frac{1}{1-\varepsilon_{2}}\right)^{k+1} \prod_{j=0}^{k+1} \max \left\{\left\|s_{k}\right\|,\left\|s_{k}\right\|^{1-p}\right\}\left\|g_{j}\right\| \cos \theta_{j},
\end{aligned}
$$

we have

$$
\prod_{j=0}^{k+1} \max \left\{\left\|s_{j}\right\|,\left\|s_{j}\right\|^{1-p}\right\}\left\|g_{j}\right\| \cos \theta_{j} \geq\left[\frac{\left(1-\varepsilon_{2}\right) c_{3} c_{2}^{2}}{\operatorname{Tr}\left(B_{0}\right)+M_{1}}\right]^{k+1} .
$$

According to Lemma 4.4 and Assumption A we know that there exists a constant $M_{2}^{\prime}>0$ such that

$$
\left\|s_{k}\right\|=\left\|x_{k+1}-x_{k}\right\| \leq\left\|x_{k+1}\right\|+\left\|x_{k}\right\| \leq 2 M_{2}^{\prime} .
$$

Combining (4.23) and (4.24), and noting that $\left\|g_{j}\right\| \cos \theta_{j}=\eta_{j}$, we get for all $k \geq 0$

$$
\prod_{j=0}^{k} \eta_{j} \geq\left[\frac{\left(1-\varepsilon_{2}\right) c_{3} c_{2}^{2}}{\left(\operatorname{Tr}\left(B_{0}\right)+M_{1}\right) \max \left\{2 M_{2}^{\prime}, 1,\left(2 M_{2}^{\prime}\right)^{1-p}\right\}}\right]^{k+1}=\epsilon_{0}^{k+1} .
$$

The proof is complete.

Now we establish the global convergence theorem for Algorithm 2.

Theorem 4.1. Let Assumption A hold and the sequence $\left\{x_{k}\right\}$ be generated by Algorithm 2. Then we have

$$
\liminf _{k \rightarrow \infty}\left\|g_{k}\right\|=0
$$

Proof. By Lemma 4.3 and (2.20), we get

$$
\begin{aligned}
f_{k+1} & \leq f\left(x_{l(k)}\right)-\varepsilon_{1}\left\|s_{k}\right\| \eta_{k} \\
& \leq f\left(x_{l(k)}\right)-\varepsilon_{1} b_{0} \min \left\{\eta_{k}^{2}, \eta_{k}^{\frac{2-p}{1-p}}\right\} .
\end{aligned}
$$

Let $t_{k}=\varepsilon_{1} b_{0} \min \left\{\eta_{k}^{2}, \eta_{k}^{\frac{2-p}{1-p}}\right\}$. By Lemma 4.5, we have

$$
\sum_{k=0}^{\infty} \min _{0 \leq j \leq n(k)} \min \left\{\eta_{k+n(k)-j}^{2}, \eta_{k+n(k)-j}^{\frac{2-p}{1-p}}\right\}<+\infty,
$$




$$
\sum_{q=1}^{\infty} \min _{0 \leq j \leq n(k)} \min \left\{\eta_{(n(k)+1) q+n(k)-j}^{2}, \eta_{(n(k)+1) q+n(k)-j}^{\frac{2-p}{1-p}}\right\}<+\infty .
$$

Denoting the sequence $\{p(q)\}$ as follows:

$$
\begin{aligned}
\min \left\{\eta_{p(q)}^{2}, \eta_{p(q)}^{\frac{2-p}{1-p}}\right\} & =\min _{0 \leq j \leq n(k)} \min \left\{H_{1 j}(q), H_{2 j}(q)\right\} \\
H_{1 j}(q) & =\eta_{(n(k)+1) q+n(k)-j}^{2} \\
q(n(k)+1) \leq p(q) & \leq(q+1) n(k)+q .
\end{aligned}
$$

Therefore

$$
\begin{aligned}
p(1)<p(2)<p(3)<\cdots<p(q-1) & <p(q)<\cdots \\
\lim _{q \rightarrow \infty} \min \left\{\eta_{p(q)}^{2}, \eta_{p(q)}^{\frac{2-p}{1-p}}\right\} & =0 \\
\lim _{q \rightarrow \infty} \eta_{p(q)} & =0
\end{aligned}
$$

which means that

$$
\lim _{k \in K} \eta_{k}=0, K \subset N
$$

where $K$ is a subset of $N=\{1,2,3, \ldots\}$. By $x_{k} \in \Omega$, and $\Omega$ is bounded, we can assume that there exists a constant $b_{3}>0$ such that $\left\|g_{k}\right\| \leq b_{3}$. Then we get

$$
\eta_{k}=\frac{-g_{k}^{T} d_{k}}{\left\|d_{k}\right\|} \leq\left\|g_{k}\right\| \leq b_{3} .
$$

We prove our theorem by contradiction. Assume that

$$
\liminf _{k \rightarrow \infty}\left\|g_{k}\right\|>0
$$

so that there exists a constant $c_{2}>0$ such that

$$
\left\|g_{k}\right\| \geq c_{2}, k=0,1,2, \ldots
$$

By Lemma 4.7, we know that there exists a constant $\epsilon_{0}$ satisfying

$$
\prod \eta_{j=0}^{k} \geq \epsilon_{0}^{k+1} \text {. }
$$

Combining (4.29) and (4.28), we deduce that for any integer $k \geq 1$,

$$
\begin{aligned}
\epsilon_{0}^{(k+1) n(k)+k} & \leq \prod_{j=1}^{(k+1) n(k)+k} \eta_{j} \\
& =\frac{1}{\eta_{0}} \prod_{q=0}^{k} \prod_{j=(n(k)+1) q}^{(q+1) n(k)+q} \eta_{j} \\
& =\frac{1}{\eta_{0}} \prod_{q=0}^{k} \prod_{0 \leq j \leq n(k)} \eta_{q(n(k)+1)+n(k)-j}
\end{aligned}
$$




$$
\begin{aligned}
& \leq \frac{1}{\eta_{0}} \prod_{q=0}^{k}\left[\eta_{p(q)} b_{3}^{n(k)}\right] \\
& =\frac{1}{\eta_{0}} b_{3}^{k n(k)} \prod_{q=0}^{k} \eta_{p(q)} .
\end{aligned}
$$

Then we have

$$
\prod_{q=0}^{k} \eta_{p(q)} \geq \eta_{0} \epsilon_{0}^{n(k)}\left[\frac{\epsilon_{0}^{n(k)+1}}{b_{3}^{n(k)}}\right]^{k} \geq\left[\frac{\epsilon_{0}^{n(k)+1}}{b_{3}^{n(k)}} \min \left\{1, \eta_{0} \epsilon_{0}^{n(k)}\right\}\right]^{k} .
$$

Using Lemma 4.6 we have

$$
\limsup _{q \rightarrow \infty} \eta_{p(q)}>0
$$

which contradicts (4.27). Therefore, we obtain

$$
\liminf _{k \rightarrow \infty}\left\|g_{k}\right\|=0 \text {. }
$$

The proof is complete.

Similar to Algorithm 2, it is not difficult to get the global convergence of Algorithm 22. Here, we only state it as follows but omit the proof.

Theorem 4.2. Let Assumption A hold and the sequence $\left\{x_{k}\right\}$ be generated by Algorithm 22. Then we have (4.25).

\section{Numerical results}

In this section, we report some numerical results on the problems [30] with initial points. All codes were written in MATLAB 7.0 and run on PC with 2.60GHz CPU processor and 256MB memory and Windows XP operation system. The parameters are chosen as: $\sigma_{1}=0.1, \sigma_{2}=0.9, \varepsilon=10^{-5}, \varepsilon_{1}=$ $0.1, \varepsilon_{2}=0.01, p=5, H=8, m_{1}=5$, and the initial matrix $B_{0}=I$ is the unit matrix.

The following Himmeblau stop rule is used [47]:

If $\left|f\left(x_{k}\right)\right|>e_{1}$, let stop $1=\frac{\left|f\left(x_{k}\right)-f\left(x_{k+1}\right)\right|}{\left|f\left(x_{k}\right)\right|}$; Otherwise, let stop $1=\mid f\left(x_{k}\right)-$ $f\left(x_{k+1}\right) \mid$.

For each problem, if $\|g(x)\|<\varepsilon$ or stop $1<e_{2}$ was satisfied, the program will be stopped, where $e_{1}=e_{2}=10^{-5}$.

Since the line search cannot always ensure the descent condition $d_{k}^{T} g_{k}<0$, uphill search direction may occur in the numerical experiments. In this case, the line search rule maybe fails. In order to avoid this case, the stepsize $\alpha_{k}$ will be accepted if the searching number is more than twenty five in line search. We also stop the program if the iteration number is more than one thousand, and the corresponding method is considered to be failed.

In Figure 1-3, "BFGS-WP-Ak1" and "BFGS-WP-Ak2" stand for the modified BFGS formula (2.8) with WWP rule and the modified BFGS formula 
(2.16) with WWP rule, respectively. "L-BFGS-A1" and "L-BFGS-A11" stand for Algorithm 1 and Algorithm 2, respectively. The detailed numerical results are listed on the web site

$$
\text { http : //210.36.16.53:8018/publication.asp?id }=33331 \text {. }
$$

Dolan and Moré [13] gave a new tool to analyze the efficiency of algorithms. They introduced the notion of a performance profile as a means to evaluate and compare the performance of the set of solvers $S$ on a test set $P$. Assuming that there exist $n_{s}$ solvers and $n_{p}$ problems, for each problem $p$ and solver $s$, they defined $t_{p, s}=$ computing time (the number of function evaluations or others) required to solve problem $p$ by solver $s$.

Requiring a baseline for comparisons, they compared the performance on problem $p$ by solver $s$ with the best performance by any solver on this problem; that is, using the performance ratio

$$
r_{p, s}=\frac{t_{p, s}}{\min \left\{t_{p, s}: s \in S\right\}}
$$

Suppose that a parameter $r_{M} \geq r_{p, s}$ for all $p, s$ is chosen, and $r_{p, s}=r_{M}$ if and only if solver $s$ does not solve problem $p$.

The performance of solver $s$ on any given problem might be of interest, but we would like to obtain an overall assessment of the performance of the solver, then they defined

$$
\rho_{s}(t)=\frac{1}{n_{p}} \operatorname{size}\left\{p \in P: r_{p, s} \leq t\right\}
$$

thus $\rho_{s}(t)$ was the probability for solver $s \in S$ that a performance ratio $r_{p, s}$ was within a factor $t \in R$ of the best possible ration. Then function $\rho_{s}$ was the (cumulative) distribution function for the performance ratio. The performance profile $\rho_{s}: R \mapsto[0,1]$ for a solver was a nondecreasing, piecewise constant function, continuous from the right at each breakpoint. The value of $\rho_{s}(1)$ was the probability that the solver would win over the rest of the solvers.

According to the above rules, we know that one solver whose performance profile plot is on top right will win over the rest of the solvers.

Figures 1,2, and 3 show that the performance of these methods is relative to $N I, N F G$, and Time, respectively, where $N I$ denotes the total number of iterations, $N F G$ denotes the total number of the function evaluations and the gradient evaluations where $N T=N F+5 N G$ (see $[6,18]$ ), and Time denotes the cpu time that these methods spent. From these three figures it is clear that the L-BFGS-A11 method has the most wins (has the highest probability of being the optimal solver).

Figure 1 shows that L-BFGS-A11 and L-BFGS-A1 outperform BFGS-WPAk1 and BFGS-WP-Ak2 about $5 \%$ and $8 \%$ test problems, respectively. The L-BFGS-A11 method is predominant among the other three methods for $t \leq 5$. 


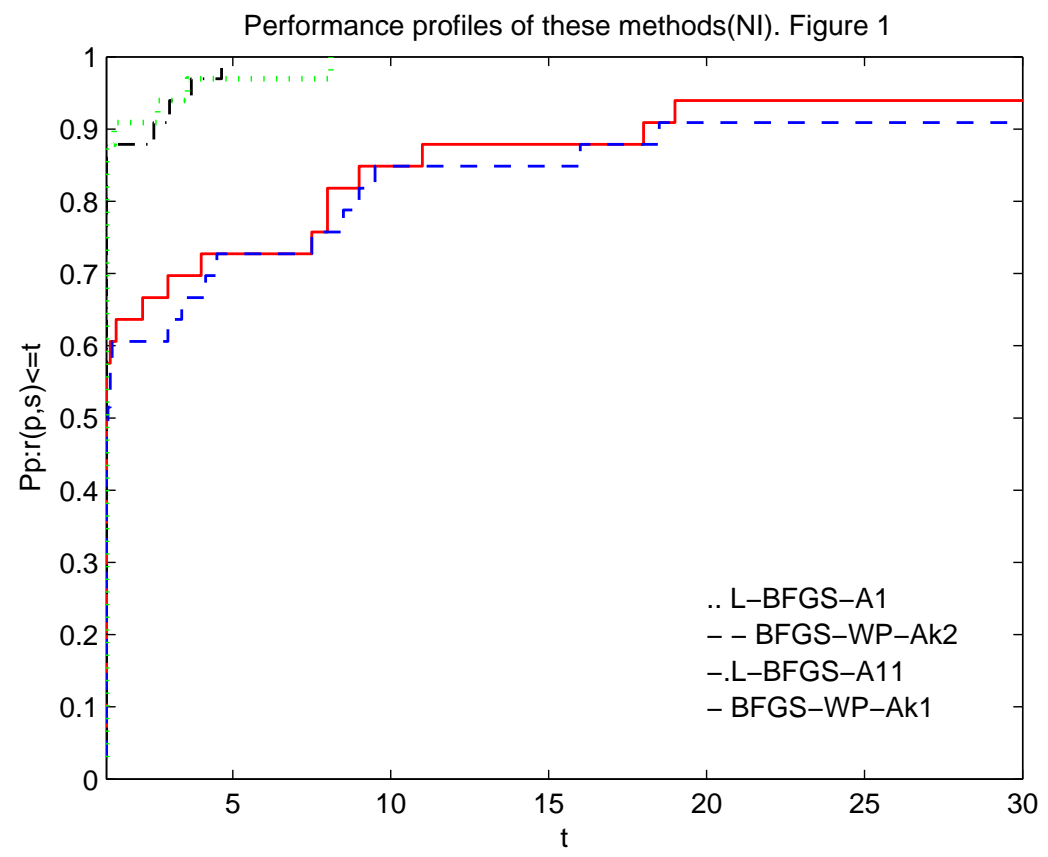

Moreover, the L-BFGS-A11 and L-BFGS-A1 and solve 100\%, and the BFGSWP-Ak1 and BFGS-WP-Ak2 method solve about $95 \%$ and $92 \%$ of the test problems successfully, respectively.

Figure 2 shows that L-BFGS-A11 and L-BFGS-A1 are superior to BFGSWP-Ak1 and BFGS-WP-Ak2 about $15 \%$ test problems. The L-BFGS-A11 and L-BFGS-A1 method can solve $100 \%$ of the test problems successfully at $t \approx 4.2$ and $t \approx 7.2$, respectively. The BFGS-WP-Ak1 and BFGS-WP-Ak2 method solve about $85 \%$ of the test problems successfully.

Figure 3 shows that L-BFGS-A11 outperforms the other three methods. The L-BFGS-A1 method and the BFGS-WP-Ak1 method solve about $95 \%$ and $91 \%$ of the test problems, respectively, and the BFGS-WP-Ak2 solves about $88 \%$ of the test problems successfully.

In summary, the presented numerical results reveal that Algorithm 1 and Algorithm 11, compared with other two methods with WWP line search and BFGS update, have potential advantages for these problems.

\section{Conclusion}

This paper gives two modified L-BFGS method with one nonmonotone line search technique for solving unconstrained optimization, which include the function value at the current and next iterative point values. The global convergence for the uniformly convex functions are established. The numerical 

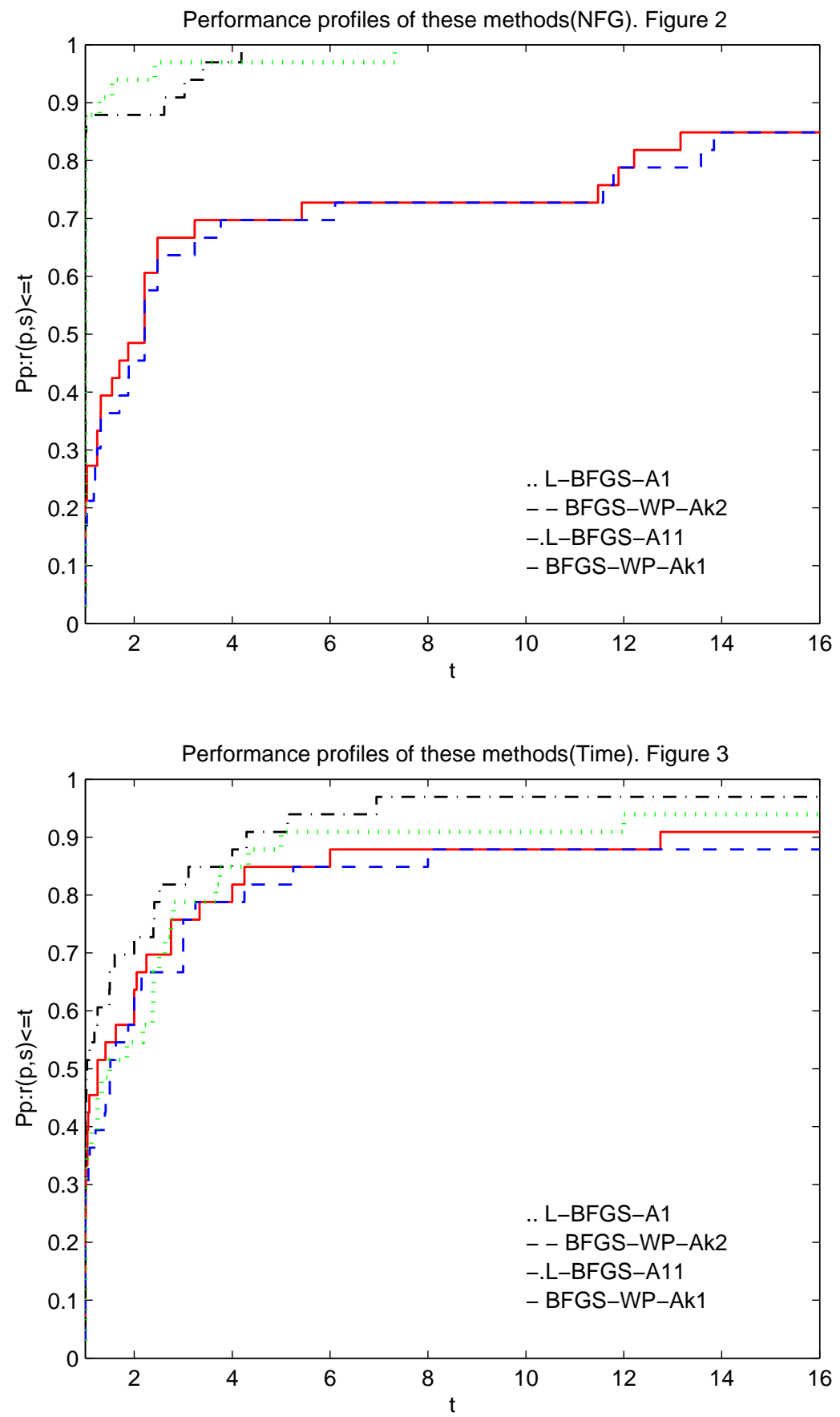
results show that the given methods are competitive to the other standard BFGS methods for the test problems.

For further research, we should study the performance of the new algorithm at different stop rules and different testing environment (such as [15]). Moreover, more numerical experiments for large practical problems should be done in the future.

\section{References}

[1] C. G. Broyden, J. E. Dennis Jr, and J. J. Moré, On the local and superlinear convergence of quasi-Newton methods, J. Inst. Math. Appl. 12 (1973), 223-245.

[2] R. H. Byrd and J. Nocedal, A tool for the analysis of quasi-Newton methods with application to unconstrained minimization, SIAM J. Numer. Anal. 26 (1989), no. 3, 727-739.

[3] R. H. Byrd, J. Nocedal, and R. B. Schnabel, Representations of quasi-Newton matrices and their use in limited memory methods, Math. Programming 63 (1994), no. 2, Ser. A, $129-156$

[4] R. Byrd, J. Nocedal, and Y. Yuan, Global convergence of a class of quasi-Newton methods on convex problems, SIAM J. Numer. Anal. 24 (1987), no. 5, 1171-1190.

[5] Y. Dai, Convergence properties of the BFGS algorithm, SIAM J. Optim. 13 (2002), no. 3, 693-701.

[6] Y. Dai and Q. Ni, Testing different conjugate gradient methods for large-scale unconstrained optimization, J. Comput. Math. 21 (2003), no. 3, 311-320.

[7] W. C. Davidon, Variable metric methods for minimization, A. E. C. Research and Development Report ANL-599, 1959.

[8] R. Dembo and T. Steihaug, Truncated Newton algorithms for large-scale unconstrained optimization, Math. Programming 26 (1983), no. 2, 190-212.

[9] J. E. Dennis Jr. and J. J. Moré, Quasi-Newton methods, motivation and theory, SIAM Rev. 19 (1977), no. 1, 46-89.

[10] - A characterization of superlinear convergence and its application to quasiNewton methods, Math. Comp. 28 (1974), 549-560.

[11] J. E. Dennis Jr. and R. B. Schnabel, Numerical Methods for Unconstrained Optimization and Nonlinear Equations, Prentice Hall Series in Computational Mathematics. Prentice Hall, Inc., Englewood Cliffs, NJ, 1983.

[12] L. C. W. Dixon, Variable metric algorithms: necessary and sufficient conditions for identical behavior of nonquadratic functions, J. Optimization Theory Appl. 10 (1972), 34-40.

[13] E. D. Dolan and J. J. Moré, Benchmarking optimization software with performance profiles, Math. Program. 91 (2002), no. 2, Ser. A, 201-213.

[14] R. Fletcher, Practical Methods of Optimization, Second edition. A Wiley-Interscience Publication. John Wiley \& Sons, Ltd., Chichester, 1987.

[15] N. I. M. Gould, D. Orban, and Ph. L. Toint, CUTEr (and SifDec), a constrained and unconstrained testing environment, revisite, ACM Transactions on Mathematical Software 29 (2003), 373-394.

[16] A. Griewank, The global convergence of partitioned BFGS on problems with convex decompositions and Lipschitzian gradients, Math. Programming 50 (1991), no. 2, (Ser. A), $141-175$.

[17] A. Griewank and Ph. L. Toint, Local convergence analysis for partitioned quasi-Newton updates, Numer. Math. 39 (1982), no. 3, 429-448.

[18] L. Grippo, F. Lamparillo, and S. Lucidi, A nonmonotone line search technique for Newton's method, SIAM J. Numer. Anal. 23 (1986), no. 4, 707-716. 
[19] _ A truncated Newton method with nonmonotone line search for unconstrained optimization, J. Optim. Theory Appl. 60 (1989), no. 3, 401-419.

[20] A class of nonmonotone stabilization methods in unconstrained optimization, Numer. Math. 59 (1991), no. 8, 779-805.

[21] J. Y. Han and G. H. Liu, Global convergence analysis of a new nonmonotone BFGS algorithm on convex objective functions, Comput. Optim. Appl. 7 (1997), no. 3, 277-289.

[22] D. Li and M. Fukushima, A modified BFGS method and its global convergence in nonconvex minimization, J. Comput. Appl. Math. 129 (2001), no. 1-2, 15-35.

[23] _ On the global convergence of the BFGS method for nonconvex unconstrained optimization problems, SIAM J. Optim. 11 (2001), no. 4, 1054-1064.

[24] G. Li, C. Tang, and Z. Wei, New conjugacy condition and related new conjugate gradient methods for unconstrained optimization, J. Comput. Appl. Math. 202 (2007), no. 2, 523-539.

[25] G. H. Liu and J. Y. Han, Notes on the general form of stepsize selection, OR and Decision Making I (1992), 619-624.

[26] _ Global convergence Analysis of the variable metric algorithm with a generalized Wolf linesearch, Technical Report, Institute of Applied Mathematics, Academia Sinica, Beijing, China, no. 029, 1993.

[27] G. H. Liu, J. Y. Han, and D. F. Sun, Global convergence of the BFGS algorithm with nonmonotone linesearch, Optimization 34 (1995), no. 2, 147-159.

[28] G. H. Liu and J. M. Peng, The convergence properties of a nonmonotonic algorithm, J. Comput. Math. 1 (1992), 65-71.

[29] W. F. Mascarenhas, The BFGS method with exact line searches fails for non-convex objective functions, Math. Program. 99 (2004), no. 1, Ser. A, 49-61.

[30] J. J. Moré, B. S. Garbow, and K. E. Hillstrome, Testing unconstrained optimization software, ACM Trans. Math. Software 7 (1981), no. 1, 17-41.

[31] S. G. Nash, A survey of truncated-Newton methods, Numerical analysis 2000, Vol. IV, Optimization and nonlinear equations. J. Comput. Appl. Math. 124 (2000), no. 1-2, $45-59$.

[32] M. J. D. Powell, On the convergence of the variable metric algorithm, J. Inst. Math. Appl. 7 (1971), 21-36.

[33] _ Some global convergence properties of a variable metric algorithm for minimization without exact line searches, Nonlinear programming (Proc. Sympos., New York, 1975), pp. 53-72. SIAM-AMS Proc., Vol. IX, Amer. Math. Soc., Providence, R. I., 1976.

[34] A new algorithm for unconstrained optimization, 1970 Nonlinear Programming (Proc. Sympos., Univ. of Wisconsin, Madison, Wis., 1970) pp. 31-65 Academic Press, New York.

[35] M. Raydan, The Barzilai and Borwein gradient method for the large scale unconstrained minimization problem, SIAM J. Optim. 7 (1997), no. 1, 26-33.

[36] J. Schropp, A note on minimization problems and multistep methods, Numer. Math. 78 (1997), no. 1, 87-101.

37] One-step and multistep procedures for constrained minimization problems, IMA J. Numer. Anal. 20 (2000), no. 1, 135-152.

[38] Ph. L. Toint, Global convergence of the partitioned BFGS algorithm for convex partially separable optimization, Math. Programming 36 (1986), no. 3, 290-306.

[39] D. J. van Wyk, Differential optimization techniques, Appl. Math. Modelling 8 (1984), no. 6, 419-424.

[40] M. N. Vrahatis, G. S. Androulakis, J. N. Lambrinos, and G. D. Magolas, A class of gradient unconstrained minimization algorithms with adaptive stepsize, J. Comput. Appl. Math. 114 (2000), no. 2, 367-386. 
[41] Z. Wei, G. Li, and L. Qi, New quasi-Newton methods for unconstrained optimization problems, Appl. Math. Comput. 175 (2006), no. 2, 1156-1188.

[42] Z. Wei, G. Yu, G. Yuan, and Z. Lian, The superlinear convergence of a modified BFGStype method for unconstrained optimization, Comput. Optim. Appl. 29 (2004), no. 3, 315-332.

[43] G. L. Yuan, Modified nonlinear conjugate gradient methods with sufficient descent property for large-scale optimization problems, Optim. Lett. 3 (2009), no. 1, 11-21.

[44] G. L. Yuan and X. W. Lu, A new line search method with trust region for unconstrained optimization, Comm. Appl. Nonlinear Anal. 15 (2008), no. 1, 35-49.

[45] _ A modified PRP conjugate gradient method, Ann. Oper. Res. 166 (2009), 73-90.

[46] G. L. Yuan, X. Lu, and Z. Wei, A conjugate gradient method with descent direction for unconstrained optimization, J. Comput. Appl. Math. 233 (2009), no. 2, 519-530.

[47] Y. Yuan and W. Sun, Theory and Methods of Optimization, Science Press of China, 1999.

[48] G. L. Yuan and Z. X. Wei, New line search methods for unconstrained optimization, J. Korean Statist. Soc. 38 (2009), no. 1, 29-39.

[49] - The superlinear convergence analysis of a nonmonotone BFGS algorithm on convex objective functions, Acta Math. Sin. (Engl. Ser.) 24 (2008), no. 1, 35-42.

[50] _ Convergence analysis of a modified BFGS method on convex minimizations, Comput. Optim. Appl. doi: 10.1007/s10589-008-9219-0.

[51] J. Z. Zhang, N. Y. Deng, and L. H. Chen, New quasi-Newton equation and related methods for unconstrained optimization, J. Optim. Theory Appl. 102 (1999), no. 1, $147-167$.

GongLin Yuan

College of Mathematics and Information Science

GuangXi University

Nanning, GuangXi, 530004, P. R. China

E-mail address: glyuan@gxu.edu.cn

ZENGXIN WeI

College of Mathematics and Information Science

GUANGXi UnivERSITY

Nanning, Guangxi, 530004, P. R. China

E-mail address: zxwei@gxu.edu.cn

YANLIN WU

College of Mathematics and Information Science

GuANGXi University

Nanning, Guangxi, 530004, P. R. China

E-mail address: wyl-kk@163.com 